\title{
Overcoming barriers to neurosurgical training in Tanzania: international exchange, curriculum development, and novel methods of resource utilization and subspecialty development
}

\author{
D. Ryan Ormond, MD, ${ }^{1}$ Joseph Kahamba, MD, ${ }^{2}$ Kevin O. Lillehei, MD, ${ }^{1}$ and \\ Nicephorus Rutabasibwa, MD²
}

1Department of Neurosurgery, University of Colorado School of Medicine, Aurora, Colorado; and 2Division of Neurosurgery, Muhimbili Orthopedic Institute, Muhimbili University of Health and Allied Sciences, Dar es Salaam, Tanzania

Tanzania sits on the Indian Ocean in East Africa and has a population of over 53 million people. While the gross domestic product has been increasing in recent years, distribution of wealth remains a problem, and challenges in the distribution of health services abound. Neurosurgery is a unique case study of this problem. The challenges facing the development of neurosurgery in Tanzania are many and varied, built largely out of the special needs of modern neurosurgery. Task shifting (training nonphysician surgical providers) and 2-tiered systems (fast-track certification of general surgeons to perform basic neurosurgical procedures) may serve some of the immediate need, but these options will not sustain the development of a comprehensive neurosurgical footprint. Ultimately, long-term solutions to the need for neurosurgical care in Tanzania can only be fulfilled by local government investment in capacity building (infrastructure and neurosurgical training), and the commitment of Tanzanians trained in neurosurgery. With this task in mind, Tanzania developed an independent neurosurgery training program in Dar es Salaam. While significant progress has been made, a number of training deficiencies remain. To address these deficiencies, the Muhimbili Orthopedic Institute (MOI) Division of Neurosurgery and the University of Colorado School of Medicine Department of Neurosurgery set up a Memorandum of Understanding in 2016. This relationship was developed with the perspective of a "collaboration of equals." Through this collaboration, faculty members and trainees from both institutions have the opportunity to participate in international exchange, join in collaborative research, experience the culture and friendship of a new country, and share scholarship through presentations and teaching. Ultimately, through this international partnership, mutual improvement in the care of the neurosurgical patient will develop, bringing programs like MOI out of isolation and obscurity. From Dar es Salaam, a center of excellence is developing to train neurosurgeons who can go well equipped throughout Tanzania to improve the care of the neurosurgical patient everywhere. The authors encourage further such exchanges to be developed between partnership training programs throughout the world, improving the scholarship, subspecialization, and teaching expertise of partner programs throughout the world.

https://thejns.org/doi/abs/10.3171/2018.7.FOCUS18239

KEYWORDS Tanzania; neurosurgical education; international exchange; residency training

$\mathrm{T}$ HE history and future of neurosurgery in Tanzania can only be fully understood through the lens of the culture, geography, and geopolitical history of this part of Sub-Saharan Africa. Tanzania is the product of the merger of Tanganyika (the mainland) and Zanzibar in 1964 following formal independence from Britain in 1961. ${ }^{3}$ Tanzania sits on the Indian Ocean in East Africa and is bordered by Burundi, the Democratic Republic of the
Congo, Kenya, Malawi, Mozambique, Rwanda, Uganda, and Zambia. Tanzania has a total land area of 945,087 sq $\mathrm{km}$ and is more than twice the size of California. It is the most populated country in East Africa with over 53 million people. Its population is skewed toward the young, given its high birth rate ( 4.8 children per woman) and high rate of HIV and other infections among the adult population. ${ }^{3}$ While Tanzania is poor in comparison to other nations,

ABBREVIATIONS COSECSA = College of Surgeons of Eastern, Central and Southern Africa; ICU = intensive care unit; LMIC = low- and middle-income country; MOI = Muhimbili Orthopedic Institute; TSS = transsphenoidal surgery; UCSOM = University of Colorado School of Medicine.

SUBMITTED May 16, 2018. ACCEPTED July 3, 2018.

INCLUDE WHEN CITING DOI: 10.3171/2018.7.FOCUS18239. 
the gross domestic product has been growing at 6\%-7\% per year for the past several years, with an annualized per capita gross domestic product of $\$ 3,300$ (US). Distribution of wealth remains a problem, however, and unemployment remains above $10 \%$, with many economic factors driving urbanization. ${ }^{3}$ Challenges in distribution of health services abound, as is true in many low- and middle-income countries (LMICs). Neurosurgery has developed sporadically in the midst of these socioeconomic factors.

Advancement toward specialized care in Tanzania began in 1971, when orthopedic and trauma services were established at the Muhimbili Hospital. ${ }^{12}$ Spine and head trauma services were originally carried out by expatriate orthopedic surgeons from Cuba, China, and the Union of Soviet Socialist Republics. However, even as the incidence of nontraumatic neurosurgical case referrals to Muhimbili increased, a number of obstacles prevented advancement of the complexity of neurosurgical care. These included the lack of operative and diagnostic facilities, subspecialty training, and continuity of service due to the lack of a permanent neurosurgeon. ${ }^{12}$ The 1980s brought change when the first Tanzanian citizens specialized in neurosurgery (trained abroad) returned to Tanzania to practice. This included pioneers such as Drs. Simpert Kinunda, Yadon M. Kohi, Peter Kadyanji, and Samwel Mlay. ${ }^{12}$ Having established practices in Dar es Salaam helped to develop neurosurgery in Tanzania's largest city and prepare the way for the current era.

The challenges facing the development of neurosurgery in Tanzania are many and varied, built largely out of the special needs of modern neurosurgery. Neurosurgery requires high-quality intensive care, specialized operative and imaging equipment, bioengineering expertise to repair and maintain specialized equipment, and well-trained allied health professionals in other important specialties such as intensive care, medicine, surgery, neurology, anesthesiology, pathology, and radiology. This has led many to conclude that surgical specialties should largely be ignored in favor of other public health priorities such as the treatment of infectious diseases, given the challenges of performing high-level subspecialty surgery in LMICs. ${ }^{4}$ However, it has been estimated that between $10 \%-25 \%$ of all deaths in LMICs could be averted with timely access to surgical care. ${ }^{4,8}$ Specifically, it has been estimated that over 400,000 deaths could be averted with the addition of subspecialty care for the following conditions alone: cataract, cleft lip and palate, congenital heart anomalies, neural tube defects, and obstetrical fistula. ${ }^{4}$ While specific numbers are not available (neurosurgery was specifically excluded from the WHO analysis in 2015), it is reasonable to believe a large burden of disease would also be averted with timely access to neurosurgical care. ${ }^{4}$

\section{Uganda as an Example of Capacity Building in East Africa}

Uganda is an interesting example of what can be achieved in a country facing similar obstacles to the development of neurosurgical capacity. Uganda is a country in East Africa, which only had 6 neurosurgeons for a population of over 33 million people as of 2015. In
March 2000, Dr. Benjamin Warf moved with his family to Mbale, Uganda, as missionaries with CURE International, opening the first pediatric neurosurgery specialty unit in Sub-Saharan Africa. Over time, surgical capacity grew, and CURE Children's Hospital of Uganda now performs over 1000 pediatric neurosurgical operations per year. ${ }^{10}$ Beginning in 2007, Dr. Michael Haglund, Professor of Neurosurgery from Duke University (North Carolina, United States), first visited Mulago Hospital at the request of a local pastor and found a 1500-bed hospital with only one intensive care unit (ICU) ventilator in the entire hospital, and operating rooms without ventilators or monitoring equipment such as pulse oximetry, automatic blood pressure cuffs, end-tidal $\mathrm{CO}_{2}$ monitors, or electrocardiogram monitoring. ${ }^{10}$ Over the next several years, with some fits and starts along the way, neurosurgical capacity increased at Mulago Hospital as well. Dr. Haglund took a 3-tiered approach: technology, twinning, and training. ${ }^{7}$ Technology has reference to helping build technological capabilities to perform neurosurgery, provide safe anesthesia, and then recover patients adequately in the recovery room, ICU, and general ward. This included not only obtaining adequate equipment but also the biomedical expertise to service and repair the equipment.? Twinning involved the concept of developing a collaborative effort between the neurosurgery department at Mulago Hospital and Duke University. ${ }^{9}$ Training began with surgical camps and then progressed to the development of a neurosurgery training program (started in 2009, with the first graduate successfully completing the College of Surgeons of Eastern, Central and Southern Africa [COSECSA] Neurosurgery Fellowship examination December 2014). ${ }^{10}$ While Uganda is far from the goal of adequate neurosurgical capacity throughout the country, they have made substantial progress toward a gradual increase in local capacity to care for the tremendous neurosurgical needs in Uganda.

\section{Madaktari Africa Experience in Tanzania}

Another example of progress toward building neurosurgical capacity in Sub-Saharan Africa has been through the efforts of Dr. Dilantha Ellegala and Madaktari Africa. ${ }^{6}$ Madaktari has implemented a "train-forward neurosurgery method" involving a formally trained neurosurgeon committing an initial 6-month time frame living and working in a local community, providing "focused education, hands-on training, research opportunities, and general advocacy for the interested MD or paramedical professional."' Through this focused effort, basic neurosurgical procedures that can be mastered in a short period of time by a nonneurosurgeon are taught to allow capacity building for a hospital previously not involved even in the most basic of neurosurgical procedures. This train-forward method has resulted in one hospital that previously performed only 18 neurosurgical procedures in 2005 now having 3 trained individuals and an average of 92 procedures per year being performed in the last 3 years of the study period. ${ }^{6}$ While this method has been criticized, claiming the use of lessskilled surgical care providers may result in poorer outcomes or less stable neurosurgical care in the long run, ${ }^{1,14}$ it has made some difference in the number of neurosurgi- 
cal procedures performed in LMICs and likely has made a difference in the lives of a number of individual patients.

\section{Current Barriers to Further Development}

Examples abound of failed efforts in LMICs, such as donated equipment sitting in warehouses without personnel available to install and maintain them in working order; children undergoing shunt placement for hydrocephalus, who then die later when their shunt gets infected or malfunctions; and the short-term medical mission surgeon who is no longer available. Task shifting (training nonphysician surgical providers) and 2-tiered systems (fast-track certification of general surgeons to perform basic neurosurgical procedures) may serve some of the immediate need for the performance of simple, life-saving neurosurgical procedures ${ }^{5,13,16}$ but will not sustain the development of a comprehensive neurosurgical footprint in an LMIC like Tanzania., ${ }^{1,14}$ Long-term surgical missions are obviously better than short-term missions, ${ }^{15,17}$ but they are often unsustainable given the limited number of neurosurgeons able to set aside the necessary time for a long-term mission and the lack of continuity and quality of training, along with significant turnover among mentors and teachers. ${ }^{14}$ Ultimately, long-term solutions to the need for neurosurgical care in Tanzania can only be fulfilled by local government investment in capacity building (infrastructure and neurosurgical training), and the commitment of Tanzanians who are invested in improving the state of neurosurgical care at their local hospitals.

\section{Potential Solutions to Current Limitations}

With this task in mind, COSECSA developed a neurosurgery fellowship curriculum and examination, approved by the governments of Ethiopia, Kenya, Malawi, Mozambique, Tanzania, Uganda, and Zimbabwe. These respective nations then set out to develop their own independent neurosurgery training programs.

\section{Muhimbili Orthopedic Institute Neurosurgery Fellowship Training Program}

Muhimbili Orthopedic Institute (MOI), Dar es Salaam, Tanzania, serves as a Department of Orthopedics and Traumatology and, combined with Muhimbili University of Health and Allied Sciences (MUHAS), has developed a residency curriculum for the formal training of neurosurgeons. The program is a total of 6 years with 3 years in general surgery and then 3 years in neurosurgery. The program has been in existence for 3 years, with its first 3 residents scheduled to graduate in August 2018, and 3 additional residents at different levels of training in the program.

\section{Current Faculty and Neurosurgery Capacity}

1) Human resources: MOI has 6 neurosurgery faculty members who are involved full time in clinical work and education;

2) Infrastructure: neurosurgery capacity at MOI has improved with the accumulation of additional qualified neu-

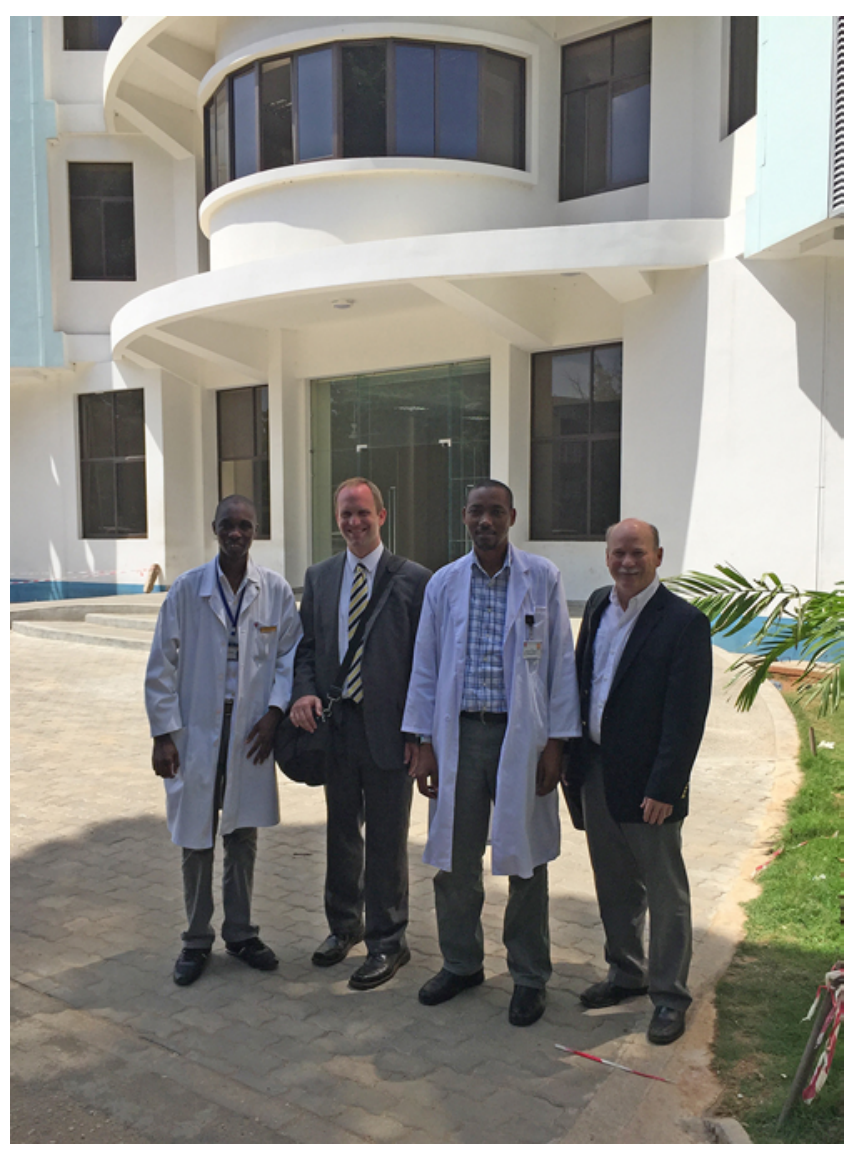

FIG. 1. Faculty members from MOI and UCSOM stand outside the partially completed new neurosurgery center, MOI, Dar es Salaam, Tanzania.

rosurgeons and international collaborations. Currently, there are 2 operating rooms, an 8-bed ICU, and a 32-bed ward. Soon, a new emergency department with increased capacity for trauma patients and a 32-bed ICU will be opened (Fig. 1). This will be augmented with a high density unit ward. MOI has also installed in-house MRI and CT machines (both with angiography capabilities) that will be operational soon (currently patients travel to other buildings for imaging studies). The expansion also will result in operating room capacity increasing to 4 rooms dedicated to neurosurgery. All these changes go hand in hand with the installation of additional monitors to enhance anesthesia and recovery room and surgical safety and also include the employment of additional biomedical engineers for equipment maintenance.

3) Spectrum of neurosurgical diseases: MOI treats a wide range of neurosurgical diseases including neurotrauma, brain tumors, neural tube defects, pediatric hydrocephalus, spinal cord tumors, spine deformities (scoliosis), and degenerative spine diseases (open and minimally invasive). However, vascular neurosurgery remains inadequately performed due to a lack of resources.

\section{Future Progress}

While significant progress has been made in the resi- 
TABLE 1. Hospital organizational structure in Tanzania

\begin{tabular}{lc}
\hline \multicolumn{1}{c}{ Facility } & Description \\
\hline Referring/consultant hospitals & $\begin{array}{c}\text { Have specialty departments and teaching clinics (currently in Dar es Salaam, Mwanza, and Moshi) } \\
\text { Regional hospitals }\end{array}$ \\
$\begin{array}{l}\text { One hospital for every } 1 \text { million population, some medical specialists, experienced general doctors, medical assis- } \\
\text { tants, schools for medical assistants }\end{array}$ \\
$\begin{array}{l}\text { One bed per } 1000 \text { inhabitants, one hospital per 100,000-200,000 population; consists of 2-4 doctors, medical as- } \\
\text { sistants, no specialty departments }\end{array}$ \\
$\begin{array}{l}\text { Neurosurgical capacity in hospitals } \\
\text { Tanzania }\end{array}$ \\
$\begin{array}{c}\text { Currently consists of the neurosurgery training program at MOI, a single neurosurgeon at Mwanza, and a small num- } \\
\text { begional, or district hospitals. The training program at MOI will deploy graduates first to referring, then to regional } \\
\text { hospitals, where they will increase neurosurgical capacity through performing surgery and teaching others to } \\
\text { perform basic procedures at district hospitals or regional hospitals without neurosurgical representation }\end{array}$ \\
\hline
\end{tabular}

dency program at MOI and other participating COSECSA programs, a number of training deficiencies remain. Quality training and preparation for the care of neurosurgical patients must be achieved for the local training of neurosurgeons to be successful, especially when considering the fact that death rates for major surgery in developing countries has been quoted at 5\%-10\%.18 Otherwise, countries should continue sending promising surgeons-in-training to quality, established programs in other countries, hoping these surgeons will return to their country after training. Unfortunately, the past has shown a significant "brain drain" when this philosophy has been implemented. Instead, improving the quality of the training experience within the home country provides a more sustainable long-term solution to the neurosurgery workforce shortage in Sub-Saharan Africa.

In a recent study conducted in Ethiopia, faculty members identified educational resources as the top perceived barrier to optimal surgical training of residents. ${ }^{2}$ Significant deficits in resident education identified by faculty included lack of knowledge on managing emergency surgical conditions and lack of disease-specific knowledge. ${ }^{2}$ Residents, in turn, reported spending an average of 12.1 hours per week on self-directed learning, suggesting that these deficits may be more related to lack of educational structure and resources than personal motivation for learning. ${ }^{2,14}$ Additionally, a survey conducted with neurosurgeons from Sub-Saharan Africa regarding perceived limitations of training demonstrated that $63 \%$ of respondents felt they had an inadequate $(35 \%)$ or no (28\%) formal training program. ${ }^{14}$ Of those with a formal training program, almost half believed their training program did not provide adequate preparation to handle incoming neurosurgical patients; this included deficiency in expertise in several subspecialties, lack of training resources and equipment, and unavailable faculty. ${ }^{14}$ Additional educational barriers noted as absent included a lack of physical resources, practical workshops, program structure, topicspecific lectures, clear goals for each level of training, supervision in the operating room, lack of proper feedback on skills, and minimal access to current innovations in the field. ${ }^{2,14}$

Sader et al. reported their belief that "the most valuable method of strengthening surgical capacity must rely on strong and resilient training programs to compensate for the lack of infrastructure."14 With this principle in mind, the MOI Division of Neurosurgery and the University of Colorado School of Medicine (UCSOM) Department of Neurosurgery set up a Memorandum of Understanding in 2016. This relationship has been developed with the perspective of a "collaboration of equals." While recognizing a significant difference in infrastructure and subspecialization, MOI neurosurgeons have been well trained in neurosurgery in other countries, and now desire to improve their curriculum and experience for neurosurgery fellows in Tanzania who will then leave Dar es Salaam to begin the process of expanding access to neurosurgery throughout Tanzania (Table 1). Our relationship is set up similar to that of the Institute of International Education, an institute set up at the end of World War I by Nobel Peace Prize winners Nicholas Murray Butler, President of Columbia University, Elihu Root, former Secretary of State of the United States, and Stephen Duggan Sr., Professor of Political Science at the College of the City of New York. The Institute of International Education was formed with the idea that international educational exchange would "incite understanding between nations." "11 Neurosurgery faculty and fellows visit for a period of 2-4 weeks. During their stay, faculty and senior fellows have the opportunity to participate in a subspecialty observership, join in collaborative research, experience the culture and friendship of a new country, and share scholarship through presentation in grand rounds and teaching and learning in hands-on courses. In between visits, collaborative research projects, online access to recorded grand rounds lectures, complex case discussions, mentoring meetings, etc., continue. Through mutual experience together, the neurosurgery division at MOI will continue to develop and progress, improving their subspecialty expertise and their ability to develop a residency program that is "strong and resilient" and compensates well for the "lack of infrastructure" in their surroundings. ${ }^{14}$ Also, as the English-language proverb "necessity is the mother of invention" implies, the infrastructure challenges in Tanzania will prove a training ground for improving neurosurgical care and residency training throughout the world.

Prior to the collaboration between MOI and UCSOM, pituitary surgery at MOI was exclusively done through craniotomy. Following a visit by Dr. Kevin Lillehei to Dar es Salaam, Dr. Nicephorus Rutabasibwa was invited to 
come to Colorado to learn the nuances of transsphenoidal surgery (TSS). This observership included time in the operating room, and hands-on work in a cadaver lab to master techniques observed in the operating room. Unfortunately, due to the challenges of credentialing foreign surgeons in the United States, the operating room experience was limited to an observership. However, a cadaver lab experience on campus added a supervised, hands-on experience during the observership, and multiple future transsphenoidal approaches with Dr. Lillehei in Dar es Salaam allowed Dr. Rutabasibwa to progress to competent surgical independence. Ultimately, by focusing the experience on a single procedure, and planning the exchange experience well, a short-term stay was made more efficacious with success more quantifiable. Since then, the practice of microscopic TSS has been instituted at MOI. This has reduced the morbidity of cranial surgery, and operative time has improved. Specifically, since this visit to Colorado, 27 pituitary adenoma resections were performed at MOI. Among these, 18 (66.7\%) were done through an endonasal, transsphenoidal microscopic approach. Three patients in the open-craniotomy group suffered a prolonged depressed level of consciousness, with a prolonged ICU stay, while in the TSS group, apart from CSF rhinorrhea, recovery has been uneventful, demonstrating an example of the tangible benefits to patient care this collaboration has already achieved.

\section{Conclusions}

Ultimately, through international partnership, mutual improvement in the care of the neurosurgical patient will develop, bringing programs like MOI out of isolation and obscurity. From Dar es Salaam, a center of excellence is developing to train neurosurgeons who can go well equipped throughout Tanzania to improve the care of the neurosurgical patient everywhere. We encourage further such exchanges to be developed between partnership training programs throughout the world, inciting "understanding between nations," and improving the scholarship, subspecialization, and teaching expertise of partner programs throughout the world.

\section{References}

1. Aliu O, Corlew SD, Heisler ME, Pannucci CJ, Chung KC: Building surgical capacity in low-resource countries: a qualitative analysis of task shifting from surgeon volunteers' perspectives. Ann Plast Surg 72:108-112, 2014

2. Cadotte DW, Blankstein M, Bekele A, Dessalegn S, Pain C, Derbew M, et al: Establishing a surgical partnership between Addis Ababa, Ethiopia, and Toronto, Canada. Can J Surg 56:E19-E23, 2013

3. Central Intelligence Agency: The World Factbook. Washington, DC: Central Intelligence Agency, 2018

4. Debas H, Donkor P, Gawande A, Jamison D, Kruk M, Mock C (eds): Essential Surgery: Disease Control Priorities, ed 3. Washington, DC: The International Bank for Reconstruction and Development/The World Bank, 2015

5. El-Fiki M: African neurosurgery, the 21st-century challenge. World Neurosurg 73:254-258, 2010

6. Ellegala DB, Simpson L, Mayegga E, Nuwas E, Samo H,
Naman N, et al: Neurosurgical capacity building in the developing world through focused training. J Neurosurg 121:1526-1532, 2014

7. Fuller A, Tran T, Muhumuza M, Haglund MM: Building neurosurgical capacity in low and middle income countries. eNeurologicalSci 3:1-6, 2015

8. Groen RS, Samai M, Petroze RT, Kamara TB, Cassidy LD, Joharifard S, et al: Household survey in Sierra Leone reveals high prevalence of surgical conditions in children. World J Surg 37:1220-1226, 2013

9. Haglund MM, Kiryabwire J, Parker S, Zomorodi A, MacLeod D, Schroeder R, et al: Surgical capacity building in Uganda through twinning, technology, and training camps. World J Surg 35:1175-1182, 2011

10. Haglund MM, Warf B, Fuller A, Freischlag K, Muhumuza $\mathrm{M}$, Ssenyonjo H, et al: Past, present, and future of neurosurgery in Uganda. Neurosurgery 80:656-661, 2017

11. Institute of International Education: History. IIE.org. (https://www.iie.org/en/Why-IIE/History) [Accessed July 31, 2018]

12. Kinasha A, Kucia EJ, Vargas J, Kavolus J, Magarik J, Ellegala DB, et al: Neurosurgery in Tanzania: a discussion of culture, socioeconomics, and humanitarians. World Neurosurg 78:31-34, 2012

13. Park BE: The African experience: a proposal to address the lack of access to neurosurgery in rural sub-Saharan Africa. World Neurosurg 73:276-279, 2010

14. Sader E, Yee P, Hodaie M: Barriers to neurosurgical training in Sub-Saharan Africa: the need for a phased approach to global surgery efforts to improve neurosurgical care. World Neurosurg 98:397-402, 2017

15. Shrime MG, Sleemi A, Ravilla TD: Charitable platforms in global surgery: a systematic review of their effectiveness, cost-effectiveness, sustainability, and role training. World J Surg 39:10-20, 2015

16. Wall AE: Ethics in global surgery. World J Surg 38:15741580,2014

17. Wilson DA, Garrett MP, Wait SD, Kucia EJ, Saguda E, Ngayomela I, et al: Expanding neurosurgical care in Northwest Tanzania: the early experience of an initiative to teach neurosurgery at Bugando Medical Centre. World Neurosurg 77:32-38, 2012

18. Yii MK, Ng KJ: Risk-adjusted surgical audit with the POSSUM scoring system in a developing country. Physiological and Operative Severity Score for the enUmeration of Mortality and morbidity. Br J Surg 89:110-113, 2002

\section{Disclosures}

Dr. Ormond received support of non-study-related clinical or research effort from Synaptive Medical.

\section{Author Contributions}

Conception and design: Ormond, Rutabasibwa. Acquisition of data: all authors. Analysis and interpretation of data: Ormond. Drafting the article: Ormond. Critically revising the article: all authors. Reviewed submitted version of manuscript: all authors. Approved the final version of the manuscript on behalf of all authors: Ormond. Administrative/technical/material support: Ormond.

\section{Correspondence}

D. Ryan Ormond: University of Colorado School of Medicine, Aurora, CO.david.ormond@ucdenver.edu. 